\title{
Specifying Interactions in Integrated Manufacturing Systems
}

\author{
David Flater \\ National Institute of Standards and Technology, U.S.A. \\ Email: dflater@nist.gov
}

Keywords Component, Integration, Modelling, Specification

\begin{abstract}
Systems of manufacturing software are often constructed by integrating preexisting software components. Accurate specification of the component interactions in these systems is needed to ensure testability and maintainability. Moreover, standards for manufacturing systems must specify the interactions to achieve interoperability and substitutability of components. In this report we discuss approaches for specifying component interactions and examine a number of potentially useful specification techniques.
\end{abstract}

\section{INTRODUCTION}

Systems of manufacturing software are often constructed by integrating pre-existing software components. The interactions between the components are central to the operation of the system, yet without a specification that unambiguously explains the expected behavior, we have only intuition to tell us whether the observed behavior is correct. STEP, known informally as the Standard for the Exchange of Product model data, has demonstrated the value of rigorously specifying data exchange interactions (for the full story, read STEP: The Grand Experience [12]). But data exchange only scratches the surface of the interactions that are possible. Even if we have complete and consistent specifications for the functions provided by two components in a system, these do not necessarily define how the components would work together to achieve a specific goal. If interoperability and substitutability of components is a goal of the specification, then the interactions must be specified completely.

In the following sections we discuss different models of interaction and summarize the features of potentially relevant tools and techniques. 


\section{INTERACTION MODELS}

\section{Nested control-flow models}

Interaction with nested flow of control [16] is a special case of synchronous communication. In these models, communication behaves like a procedure call. Borrowing terminology from the Common Object Request Broker Architecture (CORBA) [4], we would say that the component making a request remains in a blocked state until the response is complete.

Various communication infrastructures following in the footsteps of Remote Procedure Call (RPC) [23] are predisposed to the production of systems having a nested flow of control. Nested control-flow models, hereinafter referred to as nested models, enable us to analyze such systems without the complexity that would be introduced by more flexible models. The amount of nondeterminism in a nested model is limited by the fact that a component will not receive extraneous events while it is awaiting the response to a previous request.

\section{Flat control-flow models}

In systems having a flat flow of control, components are designed around a dispatch loop that never blocks. Because new requests can be processed while previous operations are still pending, the ordering of events in the system becomes more random than it would be with a nested flow of control. Whether you are using "asynchronous messaging" or "event handling" as your model, the architectural ramifications are the same. This is independent of attributes such as payload, indirection mechanisms (subscribe/notify), or support of multicasting that distinguish the various approaches to communication in a "flat" system.

The need for manufacturing systems to be responsive and deadlock-free encourages the use of a flat model. However, flat models are more difficult than nested models from a testing perspective because the actual state of any executing component no longer has a direct relationship to any distributed process that may be in progress. It is also more difficult to specify the intended behavior of event-driven systems because what we see as independent processes may run concurrently in the same component and interact in unintended ways.

The challenge for semantic modelling of flat systems is to identify the behaviors that are intended to be created by the interactions of components, rather than the behaviors of components as seen from their own limited points of view. 


\section{Mixed models}

Practical considerations sometimes get in the way of taking a purely flatmodel approach to system design. Unintended interactions between separate processes, such as competition for a shared resource, force the designer to place restrictions on the sequences of events that the system may process. Resource locking and transactions are two features that are commonly used for this purpose. The introduction of locking and transactions into a flat system can produce a system that sometimes exhibits a nested flow of control.

In the other direction, the introduction of multi-threading into a system having nested control-flow can also produce mixed behavior. A designer might use multi-threading if non-blocking transactions are the exception rather than the rule; this would be simpler than defining all of the explicit locking behavior that would need to go with a flat approach.

\section{SPECIFICATION LANGUAGES AND TECHNIQUES}

\section{Architecture Description Languages (ADLs)}

ADLs appeared in the 1990s as a promising new formalism in the software domain. However, as the cited reference describes, "There is... little consensus in the research community on what an $\mathrm{ADL}$ is, what aspects of an architecture should be modeled by an ADL, and what should be interchanged in an interchange language." [13] The point of commonality in all ADLs is support for modelling the architectural features of a software system at a high level of abstraction.

The cited reference compares ten ADLs and finds that the feature sets vary significantly. Some are designed to enable particular forms of automated analysis while others are primarily intended for abstract modelling. ADLs that enable much automated analysis necessarily have more of the flavor of a programming language or algebra than those designed only for human consumption. The kinds of automated analysis that are available with various ADLs range from deadlock detection to schedulability analysis.

Some ADLs support the modelling of system-level interactions, though it is not their primary focus. Rapide includes built-in support for modelling both synchronous and asynchronous interactions; other ADLs make use of process algebras for modelling components. Process algebras are discussed later in this document. 


\section{Component Interaction Specifications (CIS)}

While rigorously specifying the behavior of a distributed system in general is very difficult, specifying this behavior for a specific scenario is more tractable, as is demonstrated by the Component Interaction Specification (CIS) [5] based method supported by the Manufacturer's CORBA Interface Testing Toolkit (MCITT) [6], and by UML Sequence Diagrams [17] and Message Sequence Charts [21]. Unlike the other notations, CIS was designed to be translatable directly into test scaffolding for CORBA systems, but this produced disadvantages that will be discussed below.

CIS is a derivative of the integration testing method that was being used by NIST's industrial partners in the Advanced Process Control Framework Initiative (APCFI) [19]. This method, in turn, made use of ideas that are also used in UML Collaboration Diagrams [18].

A CIS interaction scenario consists of a tree of requests having specified inputs, outputs, and/or return values. The tree is rooted at a test client that initiates the entire chain of events. In order to capture the tree structure of the interactions in a flat ASCII script, an outline numbering convention similar to that of UML Collaboration Diagrams is used.

CIS assumes a nested flow of control for interactions. For CORBAbased systems having a nested flow of control, CIS is a simple and powerful tool. It enables automatic generation of run-time assertions to verify that the inputs and returns for each interaction are as specified in an actual running system. Unfortunately, although the CIS syntax is expressive enough to describe an entire tree of interactions through a distributed system, it assumes a single source of activity. To remove this limitation from the CIS syntax would be easy, but removing it from MCITT's test code generation would require a switch to a more intrusive testing approach.

\section{Finite state machines}

Finite state machines (FSMs) are simple abstractions of component behavior comprised of a set of states and transitions between them, with defined criteria for triggering the transitions.

FSMs are the cornerstone of many different specification languages and techniques. For example, Specification and Description Language (SDL) [20], a standard of the International Telecommunication Union (ITU), is now used by a popular software product for realtime modelling, simulation, and analysis. Some impressive software products for FSM analysis are also available free from universities and research laboratories. 
Like process algebras (below), finite state machines are a high-level abstraction. They provide a straightforward way to model and analyze networking protocols, embedded control, and other algorithms that lend themselves to finite state analysis without unnecessary implementation detail. The tool support for finite state analysis is very good, enabling properties of systems to be proven or refuted automatically.

With FSM approaches, the focus is on specifying the behavior of individual components, rather than on specifying interactions directly. However, given a complete set of FSMs, all possible interactions and emergent system behaviors can be deduced. They are therefore an efficient tool for modelling systems with flat control-flow, where the interactions of components having simple state spaces give rise to countless possible behaviors depending on the interleaving of events.

FSMs are less useful for applications having a complicated flow of control because the task of identifying finite state spaces for the components of the system becomes complex and error-prone. The most frequently cited reason for failure of finite state analysis is an explosion in the size of the state space resulting from an attempt to model a complex system.

\section{Process algebras}

A process algebra is a formal language for specifying and reasoning about the behavior of interacting processes. Many process algebras exist; here we will discuss only a few prominent examples.

Communicating Sequential Processes (CSP) [8] was published in 1978 in Communications of the ACM by C.A.R. Hoare. Since then it has been used as the basis for several parallel programming and specification languages. Various software exists for simulating and/or checking CSP specifications for properties such as deadlock freeness.

Milner's Calculus of Communicating Systems (CCS) [14] was emerging around the same time and also became the inspiration for much later work. Both CSP and CCS, in their original forms, assume that processes synchronize on communication, so the domains of systems that they can model are basically the same. However, they have subtle semantic differences 0 .

Language of Temporal Ordering Specification (LOTOS) [10] is an International Organization for Standardization (ISO) standard which was used in the Open Systems Interconnection (OSI) project [11]. It inherits some ideas from both CSP and CCS and is generally preferred for formal specification and verification of networking protocols.

A process algebra can help to separate the essence of an interactiondriven system from the details of any given interaction. Using process 
algebras, it is sometimes possible to construct formal proofs of properties of distributed systems. In cases where the pattern of interactions between software components becomes complex, process algebras can be employed to analyze or even define the specification at a high level of abstraction.

Different process algebras vary in their capacity for modelling time and synchronization, and the level of software support for using different algebras varies as well. Given an algebra with the correct feature set, it is possible to model nested, flat, or mixed control flows.

In general, process algebras permit more direct modelling of communication and synchronization than is possible with FSM-based techniques.

\section{Unified Modelling Language (UML)}

UML is really a collection of modelling languages and techniques that have been harmonized with one another and bundled under a common name. It began as a unification of object models, but quickly expanded to enable modelling of many different aspects of a software system. With the support of the Object Management Group and leading software firms, UML has become established as the canonical language for software models.

UML encompasses Static Structure Diagrams, Use Case Diagrams, Sequence Diagrams, Collaboration Diagrams, Statechart Diagrams, Activity Diagrams, and Implementation Diagrams, as well as the recently canonized Object Constraint Language (OCL). OCL first appeared under that name in 1997 as part of a joint IBM and ObjecTime Ltd. response to the firs1 Analysis and Design RFP [9][15]. OCL appears to have evolved at IBM from the Integrated Business Engineering Language (IBEL) and/or the Syntropy method [3]. Other parts of UML are easily recognizable as evolved and assimilated versions of popular, pre-existing computer science abstractions, including Entity-Relationship Diagrams [2], Harel Statecharts [7], Petri Nets [22], and classical flowcharts.

Such an assemblage of modelling techniques clearly aspires to provide every feature that could reasonably be needed. Nevertheless, UML is constantly being extended. Because the UML core has achieved a "critical mass" of industrial usage, it is generally easier to communicate using ar extension to UML than using a completely different language.

For specifying interactions, the most applicable part of UML is the Sequence Diagram, which provides sufficient syntax and semantics to mode. flat, nested, or mixed control flows. There is even a software product that can verify whether the interactions in a simulated system conform to : Sequence Diagram. The syntax is easily extended to include special cases of control flow, such as balking and time-outs, that are not handled by mos 
modelling techniques. These extensions are not understood by automated tools, but they are valuable for specifications that could not be expressed using a more formal, more restrictive notation.

\section{SUMMARY}

Having reviewed the state of the art in techniques for specifying interaction-driven systems, we find that there are many ways to do it, but always with a tradeoff between rigor and flexibility or ease-of-use. For a system having difficult kinds of interactions, one can obtain a precise characterization of a simplified view of the system that may not be accurate, or a less formal characterization of the system in all of its complexity that may prove to be insufficiently precise or incomplete.

The Unified Modelling Language has achieved a "critical mass" of usage. Although some tools use it in a rigorous way, UML favors flexibility and ease-of-use. In some contexts, we are obliged to sacrifice ease-of-use to achieve a level of rigor that is appropriate to the task at hand. This paper has discussed the different methods and tools that are available to meet different requirements, and the reader is encouraged to choose wisely for his or her own context.

\section{REFERENCES}

[1] Stephen D. Brookes, "On the relationship of CCS and CSP," in Lecture Notes in Computer Science \#154: Automata, Languages, and Programming, 10 Colloquium, Springer-Verlag, 1983, pp. 83-96.

[2] Peter P. Chen, "The Entity-Relationship Model - Toward a Unified View of Data," ACM Transactions on Database Systems, vol. 1, no. 1, 1976.

[3] Steve Cook and John Daniels, Designing Object Systems: Object-Oriented Modelling with Syntropy, Prentice Hall, 1994.

[4] CORBA/IIOP 2.3.1 Specification, http://www.omg.org/cgi-bin/doc?formal/99-10-07, 1999.

[5] David Flater, Component Interaction Specifications, http://www.mel.nist.gov/msidstaff/flater/mcitt/cis.html, 1998.

[6] David Flater, MCITT home page, http://www.mel.nist.gov/msidstaff/flater/mcitt/, 1999.

[7] David Harel, "Statecharts: a visual formalism for complex systems," Science of Computer Programming, vol. 8, 1987, pp. 231-274.

[8] C.A.R. Hoare, "Communicating Sequential Processes," Communications of the ACM, Volume 21, Number 8, August 1978, pp. 666-677.

[9] IBM/ObjecTime Ltd. joint submission on AD RFP1, http://www.omg.org/cgibin/doc?ad/97-01-18, 1997.

[10] ISO 8807:1989, Information processing systems - Open System Interconnection LOTOS - A formal description technique based on the temporal ordering of observational behaviour. Available from ISO, http://www.iso.ch/. 
[11] ISO/IEC 7498, Information technology - Open Systems Interconnection - Basic Reference Model. Available from ISO, http://www.iso.ch/.

[12] Sharon J. Kemmerer, editor, STEP: The Grand Experience, NIST Special Publication \#939, U.S. Government Printing Office, Washington, D.C., 1999. Available at http://www.mel.nist.gov/msidlibrary/summary/9920.html.

[13] Nenad Medvidovic and Richard N. Taylor, "A Classification and Comparison Framework for Software Architecture Description Languages," IEEE Transactions on Software Engineering, Vol. 26, No. 1, January 2000, pp. 70-93.

[14] Robin Milner, Lecture Notes in Computer Science \#92: A Calculus of Communicating Systems, Springer-Verlag, 1980.

[15] Object Analysis and Design PTF - RFP1, http://www.omg.org/cgi-bin/doc?ad/96-0501, 1996.

[16] The concepts "nested flow of control" and "flat flow of control" as applied to interactions are canonized in OMG Unified Modelling Language Specification version 1.3, http://www.omg.org/cgi-bin/doc?ad/99-06-08, 1999, Section 3.62, "Message and Stimulus."

[17] Ibid., Notation Guide, Part 7, Section 3.59: Sequence Diagram.

[18] Ibid., Notation Guide, Part 8, Section 3.65: Collaboration Diagram.

[19] Project Brief: Advanced Process Control Framework Initiative, http://jazz.nist.gov/atpcf/prjbriefs/prjbrief.cfm?ProjectNumber=95-12-0027, National Institute of Standards and Technology, 1996.

[20] Recommendation Z.100 (03/93) - CCITT specification and description language (SDL). Available from ITU, http://www.itu.int/.

[21] Recommendation Z.120 (10/96) - Message Sequence Charts (MSC). Available from ITU, http://www.itu.int/.

[22] W. Reisig, Petri Nets: An Introduction, Springer-Verlag, 1985.

[23] RFC 1831, "RPC: Remote Procedure Call Protocol Specification Version 2," http://www.faqs.org/rfcs/rfc1831.html, 1995. 